\title{
Forschung braucht Ethik
}

\section{Soziale Arbeit muss bei der Gewinnung neuer Erkenntnisse besonders sensibel sein}

\author{
Andreas Lienkamp
}

Prof. Dr. Andreas Lienkamp ist Hochschullehrer für Theologische Ethik an der Katholischen Hochschule für Sozialwesen Berlin, Gründungsmitglied des Berliner Instituts für christliche Ethik und Politik sowie Privatdozent für

Christliche Sozialethik an der Universität Bamberg.

E-Mail lienkamp@khsb-berlin.de
Forschung ist ein ambivalentes, aber gleichwohl unverzichtbares Unterfangen. Doch gerade bei der Gewinnung neuer Erkenntnisse in Theorie und Praxis Sozialer Arbeit müssen die Bewahrung und $\mathrm{Hu}$ manisierung menschlichen Lebens und seiner Umwelt im Mittelpunkt stehen.

Forschung als das methodisch-systematische, wissenschaftliche Bemühen um Erkenntnis zählt zu den zentralen Aufgaben der Hochschulen, somit auch zum Auftrag von Fachhochschulen für Soziale Arbeit. Zu deren Forschungsprofil gehören sowohl die angewandte Forschung zur Lösung individueller Notlagen und sozialer Probleme als auch die Grundlagenforschung im Sinne der theoretischen Beschäftigung mit den Voraussetzungen der einschlägigen Fach- und Bezugsdisziplinen. Forschung im Bereich Sozialwesen findet aber nicht nur an Hochschulen, sondern als Praxisbegleitforschung auch in den vielfältigen Handlungsfeldern Sozialer Arbeit statt.

Da sich die Wissenschaften der Sozialen Arbeit selbst als normative Handlungswissenschaften verstehen, gehört die Ethik ins Zentrum dieser Disziplinen. Ja, ihr kommt gegenüber Theorie und Praxis der Primat zu. Verstehen wir mit Dietmar Mieth Ethik als das gemeinsame Nachdenken über strittige Fragen der Moral, und Moral als das Gemenge aus Werten, Normen und Haltungen, die sich wiederum in Institutionen und Strukturen verfestigen (können), dann ist deutlich, dass das Gebiet der Forschung einer eigenen Ethik bedarf, denn es geht auch hier um strittige Fragen der Moral, deren ethische Reflexion somit zum Kerngeschäft einer sich ihrer Verantwortung stellenden Sozialen Arbeit gehört.

Von der Wortherkunft her bedeutet »forschen « so viel wie »nach etwas fragen «. Wenn Menschen in einer förderlichen Entwicklungsumgebung aufwachsen, fragen sie von klein auf nach dem Wieso, Weshalb und Warum. Somit kann der forschende Umgang mit der Wirklichkeit als eine anthropologische Grundgegebenheit betrachtet werden. Schon für Aristoteles gehörte das Streben nach Wissen zum Wesen des Menschen. Neugierde und Wissensdurst sind dem vernunftbegabten »Mängelwesen « Homo sapiens offensichtlich in besonderer Weise in die Wiege gelegt. Sie waren und sind Antrieb für Wissenschaft und Technik sowie Motor der neuzeitlichen Fortschrittsidee.

Forschungsfreiheit als das Recht, sich ungehindert wissenschaftlich forschend betätigen zu können, wurde in der zweiten Hälfte des 20. Jahrhunderts in den Rang eines Menschenrechts erhoben. So betont Papst Johannes XXIII. in seinem Weltrundschreiben Pacem in terris von 1963 - noch vor den Vereinten Nationen - »das Recht, frei nach der Wahrheit zu forschen « (Ziffer 29). Drei Jahre darauf verpflichten sich die Vertragsstaaten des Internationalen Paktes über wirtschaftliche, soziale und kulturelle Rechte, die »zu wissenschaftlicher Forschung und schöpferischer Tätigkeit unerlässliche Freiheit zu achten « (Art. 15 III). Bereits 1949 fand der Gedanke der Forschungsfreiheit nach ihrer massiven Unterdrückung während des Nationalsozialismus - Eingang in das deutsche Grundgesetz: »Kunst und Wissenschaft, Forschung und Lehre sind frei «, heißt es in Artikel 5 III lapidar. Die Ausübung der Rechte und Freiheiten unterliegt allerdings den üblichen Schranken, muss also jeweils mit den Rechten und Freiheiten Anderer, insbesondere der unmittelbar »Beforschten «, sowie dem Gemeinwohl abgewogen werden.

Spätestens mit den unter nationalsozialistischer Regie, auch im Interesse von Wissenschaft und Wirtschaft, durchgeführten rücksichtslosen Menschenversuchen hat Forschung ihre vermeintliche Unschuld verloren. Forschung zeigt hier ihr zweites, hässliches Gesicht. Die historischen Erfahrungen führten auch international zu einem Umdenken, das seinen Niederschlag fand u. a. im Nürnberger 
Kodex von 1946, im Genfer Ärztegelöbnis von 1948 sowie in der Deklaration des Weltärztebundes zu »Ethischen Grundsätzen für die medizinische Forschung am Menschen" aus dem Jahr 1966.

Schon Thomas von Aquin hatte zu bedenken gegeben, dass sich das Streben nach Erkenntnis mit sittlichen Verfehlungen vermischen könne. »Die Wahrheitserkenntnis ist an sich etwas Gutes. Wegen gewisser Umstände jedoch kann sie schlecht werden, z. B. wegen etwaiger Folgen oder wenn sich jemand wegen seiner Kenntnisse aufbläht ... oder wenn der Mensch seine Kenntnisse zum Sündigen ", also für egoistische Zwecke, »missbraucht « (S.th. II II q. 167).

Darüber hinaus ist Forschung nolens volens mit Folgen kürzerer und längerer Reichweite verbunden. Sie kann großen Nutzen stiften, aber auch verheerenden Schaden anrichten, wobei die Vor- und Nachteile höchst ungleich verteilt sein können. Kernelemente eines jeden Forschungsprojektes sind somit eine verantwortlich durchgeführte Güterabwägung und Forschungsfolgenabschätzung, die es auch rechtlich zu implementieren und institutionell, etwa in starken und unabhängigen Ethik-Kommissionen, zu verankern gilt.

Bei der Prüfung von Forschungsvorhaben ist der Grundsatz der Verhältnismäßigkeit der Mittel hilfreich. Demnach muss nicht nur das Forschungsziel als legitim ausgewiesen werden. Darüber hinaus muss bei allen Projekten neben ihrer Geeignetheit zur Erreichung dieses Zwecks auch ihre Erforderlichkeit gegeben sein, das heißt, es darf unter den methodischen Alternativen kein Verfahren geben, das gleichwertig und zugleich "milder ", also weniger kostenträchtig oder risikobehaftet ist. Abschließend muss noch die Angemessenheit geprüft werden, ob also der erwartete Nutzen den möglichen Schaden des Vorhabens in hinreichendem Maße übersteigt.

Dabei ist aus menschenrechtlicher Sicht eine rein utilitaristische Betrachtungsweise abzulehnen, denn grundlegende Werte wie die Menschenwürde - und sei es eines einzigen Individuums - dürfen auch dann nicht missachtet werden, wenn die Nutzenbilanz des Projekts insgesamt positiv erscheint. Eine Unschuldige, ein Unschuldiger darf nicht für noch so viele Andere geopfert werden. Damit

würde sie oder er zum bloßen Objekt degradiert - ein Verstoß gegen ihre bzw. seine Menschenwürde, den schon der kategorische Imperativ Immanuel Kants verbietet.

Für kein oder zumindest kaum ein menschliches Unterfangen gibt es hinsichtlich seiner Wirkungen und seines Erfolgs eine hundertprozentige Sicherheit. Das Ideal vollständiger Information über alle, auch langfristige Folgen und Nebenfolgen von Handlungen und Maßnahmen bleibt aufgrund der zur condition humaine essentiell gehörenden Endlichkeit ein unerreichbares Ziel. Zudem kann die Reversibilität möglicher negativer Forschungsfolgen nicht garantiert werden. Deshalb sollte hier das Vorsichtsprinzip

\section{"Ethische Reflexionen gehören zum Kerngeschäft der Sozialen Arbeit «}

walten. Besonders riskante Unternehmen sollten entweder nur unter hinreichenden Sicherheitsvorkehrungen durchgeführt werden oder ganz unterbleiben. Das gilt insbesondere, wenn konditionale Güter, wie das Leben oder die Gesundheit, verletzt werden könnten; Güter, die die Bedingung der Möglichkeit zur Wahrnehmung einer Reihe oder sogar aller anderen wichtigen Güter sind. Die Beweislast für die Unbedenklichkeit eines Vorhabens liegt dabei aufseiten der Forschenden.

Je verwundbarer die zu beforschenden Menschen sind, je weniger resilient, desto mehr Vorsicht ist geboten. Gegenüber Personen etwa, die nur über einen geringen Bildungsstand verfügen, sozial benachteiligten Schichten, Minderheiten oder Randgruppen angehören, was auf viele Adressatinnen und Adressaten Sozialer Arbeit zutrifft, ist besondere Aufmerksamkeit gefordert. Unter noch größerem Vorbehalt und Rechtfertigungszwang steht die Forschung an Nicht-Einwilligungsfähigen, etwa an Kindern oder an Menschen mit (schwerer) geistiger Behinderung, Demenz oder psychischer Erkrankung.

Cui prodest? - wem nützt es? - die alte ideologiekritische Frage ist bei jedem Projekt zu stellen. Denn Erkenntnis und Interesse sind - dies hat Jürgen Habermas hinreichend belegt - nicht voneinander zu trennen. Forschung ist nie interesselos.
Ohne Interesse gäbe es weder Wissenschaft noch Forschung. Was angezielt werden sollte, ist darum nicht eine letztlich unerreichbare Objektivität, sondern die ehrliche Selbstreflexion und anschließende Offenlegung der leitenden subjektiven Interessen. Wertfreiheit ist eine wirklichkeitsfremde Illusion. Werte begleiten vielmehr notwendig jeden Forschungsprozess.

Der Ethik-Kodex der US-amerikanischen National Association of Social Workers aus dem Jahr 2008 enthält ähnlich wie der britische Code of Ethics von 2002 (Kapitel 4.4.4) - einen eigenen Abschnitt zum Thema »Evaluation and Research « (5.02). Darin verpflichten sich die Mitglieder, Forschung zu unterstützen und zu ermöglichen, um damit ihrerseits zur Weiterentwicklung des Wissens beizutragen. Als Forschende wollen sie mögliche Folgen ihres Handelns bedenken sowie den Schutz von Probandinnen und Probanden sicherstellen: den Schutz ihrer Würde, ihrer Privatsphäre und ihres Wohlergehens. Allerdings kann es zu dilemmatischen Situationen kommen, etwa wenn das Mandat zum Schutz des Kindeswohls mit der Pflicht zur Wahrung der Anonymität in Konflikt gerät.

Forschung an Menschen darf, das fordern beide Kodizes, nur mit deren völlig freiwilliger und ausdrücklicher (schriftlicher) Einwilligung und nur nach sachgerechter und verständlicher sowie zu dokumentierender Aufklärung über Zweck, Verfahren und Dauer, über Vorteile, aber vor allem über mögliche Belastungen, Risiken und Nebenwirkungen erfolgen. Darüber hinaus muss über die Rechte der Versuchspersonen (u. a. auf hoch professionelle Durchführung lege artis, auf jederzeit mögliche, nachteilsfreie Verweigerung der Mitwirkung sowie auf Schutz der personenbezogenen Daten und der Privatsphäre), über Sicherheitsmaßnahmen, die Herkunft der Forschungsmittel, die institutionelle Einbettung der Forschenden, mögliche Rollen- und Interessenkonflikte, die weitere Verwendung der Ergebnisse und den Zugang zu ihnen informiert werden; auch wenn eine solche 
Informationspolitik die Gewinnung von Probandinnen und Probanden erschwert oder gar unmöglich macht. Dies ist der Preis, den eine Soziale Arbeit, die sich als Menschenrechtsprofession definiert, zu zahlen hat.

Es können sich allerdings weitere Zielkonflikte ergeben. Was ist beispielsweise, wenn eine umfassende Aufklärung aus forschungsstrategischen Überlegungen unterbleiben soll, etwa weil dadurch die Ergebnisse in einer Weise beeinflusst würden, die diese unbrauchbar machte? Ein Ausweg wäre, nachträglich die Einwilligung in die Weiterverwendung des erhobenen Materials einzuholen, wie die Deutsche Gesellschaft für Erziehungswissenschaft in ihrem Ethik-Kodex von 1999 vorschlägt ( $\mathbb{4}$ II). Darf man aber Versuchspersonen heimlich beobachten oder hinters Licht führen, um von ihnen Informationen zu erhalten oder bei ihnen Verhaltensweisen zu provozieren, die sie vollständig aufgeklärt - nicht preisgeben oder an den Tag legen würden? Heiligt der gute Zweck hier die Mittel? Die Kritik am Milgram-Experiment hat genau dies und zu Recht bestritten. Aber andererseits würden uns wichtige Erkenntnisse dann nicht zur Verfügung stehen. $\mathrm{Ob}$ auf die "ganze Wahrheit « gegenüber den Forschungsteilnehmenden verzichtet werden kann, sollte im konkreten Fall von einer Ethik-Kommission beurteilt werden.

Die geforderte völlige Freiwilligkeit gebietet es, keinen wie auch immer gearteten Druck auf diejenigen Personen auszuüben, die man zur Forschungsteilnahme gewinnen will. Solcher Druck kann in der sanften Überredung intellektuell Unterlegener bestehen sowie aus Macht- oder Abhängigkeitsverhältnissen resultieren. Selbst positive Anreize können die Autonomie der Entscheidung zur Mitwirkung gefährden, weil hierdurch, gewollt oder nicht, prekäre Lebenslagen ausgenutzt werden können. Wird etwa eine finanzielle Vergütung geboten, kann dies bedürftige Personen dazu veranlassen, entgegen ihren eigentlichen Vorbehalten in eine riskante Teilnahme einzuwilligen.

Grundlage des wissenschaftlichen Ethos' ist eine selbstkritische, revisionsbereite und wahrheitsgemäße Haltung zur eigenen Forschung, ihren Theorien, Methoden und Resultaten. Die Versuchung ist groß, die eigenen Ergebnisse gegenüber der Öffentlichkeit, der scientific community und den Geldgebern aufzu- werten oder gar überzubewerten, will man im Wissenschaftsbetrieb Karriere machen oder schlicht weiter im Geschäft bleiben. Da solche unerwünschten Anreize existieren, gilt es, über die vorhandene wissenschaftliche Begutachtung und die bestehenden rechtlichen Rahmenbedingungen hinaus, schon unter Studierenden ein Bewusstsein für die Unverzichtbarkeit wissenschaftlicher Redlichkeit zu stärken. Zum Forschungsethos gehört, in internen Berichten und öffentlich zugänglichen $\mathrm{Pu}$ blikationen keine relevanten mitteilbaren Erkenntnisse zu unterschlagen sowie zwischen ungeprüften Hypothesen, gut begründeten Annahmen und bewiesenen Aussagen klar erkennbar zu unterscheiden. Nicht zuletzt zählen Nachvollziehbarkeit, Überprüfbarkeit und Verständlichkeit zu den leider oftmals missachteten Normen der Forschungsethik.

\section{Grundsätze}

In Deutschland verpflichten sich auf dringende Empfehlung der Deutschen Forschungsgemeinschaft immer mehr Hochschulen inzwischen auf »Grundsätze guter wissenschaftlicher Praxis sowie Regeln und Verfahren zu deren Sicherung und für den Umgang mit wissenschaftlichem Fehlverhalten «, die es jedoch stärker als bisher von allen Beteiligten zu implementieren, kommunizieren, konkretisieren und natürlich vor allem zu befolgen gilt.

In Zeiten, in denen aufgrund anhaltender öffentlicher Finanzknappheit Drittmittel für die Ermöglichung von Forschung, aber auch für die Reputation der Hochschulen und ihres akademischen Personals immer größere Bedeutung erlangen, wächst die Gefahr, dass sich Forschende Auftraggebern und ihren Zielen unterordnen. Dazu gehört die nachträgliche Legitimationsbeschaffung für längst schon gefällte unliebsame Entscheidungen, die vom Geldgeber nun als »wissenschaftlich « begründet präsentiert werden können. Nicht selten wird dabei - zum Teil durchaus vermeidbar - auch die Entscheidung über die Veröffentlichung der Forschungsergebnisse vertraglich an den Auftraggeber abgetreten. Dieser kann dann für ihn unvorteilhafte Erkenntnisse zurückhalten oder uminterpretieren, ohne dass die Urheber legal darauf Einfluss nehmen könnten. Bei besonders gravierenden Ergebnissen stellt sich darum die Frage nach der Berechtigung oder sogar Verpflichtung zur eigentlich nicht erlaubten Weitergabe von Informationen (Whistle-Blowing), verstanden als Ultima Ratio, wenn alle anderen Wege erfolglos bleiben.

Ein weiterer ethischer Aspekt ist der Umgang mit Erkenntnissen aus früheren Forschungen, deren Theorien oder Methoden nach heutigen Maßstäben fragwürdig erscheinen oder sogar eindeutig zu verurteilen sind. Dürfen so gewonnene Resultate verwendet werden, auch wenn dies wie eine nachträgliche Legitimierung der damals angewandten Praktiken ausgelegt werden kann? Darf man den Opfern eine posthume Zustimmung einfach unterstellen? Immerhin könnten die Resultate Nachgeborenen nützen, so dass das frühere Leid weniger sinnlos erscheint. Hier ist im Einzelfall nach dem Schweregrad der Verletzung von Rechten zu prüfen, ob dieses Argument trägt.

Ein zusätzliches Problem ist die Abwägung zwischen dem Recht auf Wissen und dem entgegenstehenden Recht auf Nichtwissen. Biografie-Forschung beispielsweise ist eine Methode, bei der das Dilemma besonders offensichtlich ist. Als Forschender kann ich letztlich nicht absolut sicher einschätzen, was meine Fragen beim Gegenüber auslösen, welche aus Gründen des Überlebenkönnens verdrängten traumatischen Erlebnisse ich mit meinem Interview möglicherweise wieder ins Bewusstsein hebe. Hier werden mitunter - nicht selten leichtfertig - Menschenversuche mit ungewissem Ausgang unternommen.

Forschung, so mein Fazit, ist ein ambivalentes, aber gleichwohl unverzichtbares Unterfangen, auch in den Professionen der Sozialen Arbeit und ihren wissenschaftlichen Disziplinen, wenn sie denn ihr Wissen erweitern und wirksam sein wollen.

Normative Reflexionen in Gestalt einer menschenrechtlich und verantwortungsethisch ausgerichteten Forschungsethik sind aber ebenso unverzichtbar. Sie können über offene und versteckte Problemlagen im Forschungsprozess aufklären, für die Chancen und Risiken sowie die auf dem Spiel stehenden Werte rechtzeitig sensibilisieren $-u$. a. indem sie das geplante Forschungsvorhaben vorab aus dem Blickwinkel der besonders Bedürftigen und Gefährdeten betrachten - und damit zu einer Forschungspraxis anleiten, die sich, auch mittels berufsethischer 
Standards, in verantworteter Autonomie und um der Rechte der Anderen und ihrer Würde willen selbst Regeln unterwirft und entsprechende gesellschaftlich formulierte rechtliche Schranken nicht nur toleriert, sondern bejaht und unterstützt, ohne dabei die persönliche Verantwortung zu suspendieren.

Als ethisches Leitprinzip jeder sozialprofessionellen Forschung legt sich dabei
- auch gemäß der normativen Selbstbestimmung Sozialer Arbeit - die »Bewahrung und Humanisierung des menschlichen Lebens samt seiner vielfältigen Umwelt « (Otfried Höffe) nahe.

\section{Forschung in der Sozialen Arbeit}

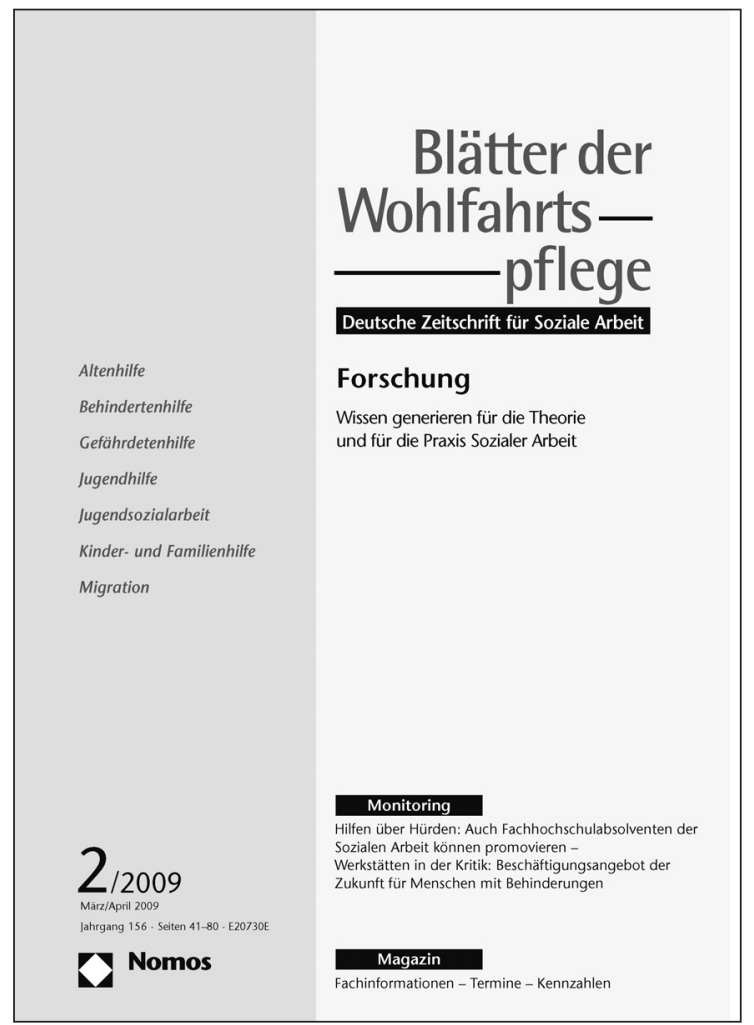

Jedes Fachgebiet ist auf der Suche nach neuen Erkenntnissen und nach besseren Methoden. Forschung ist denn auch eine der Voraussetzungen, damit eine Disziplin als eigenständige Wissenschaft gelten kann, wie Bernd Birgmeier und Martin Stummbaum in ihrem Beitrag zu einem Themenheft der Blätter der Wohlfahrtspflege über Forschung in der Sozialen Arbeit darlegen. Die Soziale Arbeit hat auf dem Weg zu einer Sozialarbeitswissenschaft diese Bedingung erkannt und begonnen, ihre Forschung zu systematisieren, zu intensivieren und zu reflektieren. Anwendungsbezogene Studien gibt es an den Ausbildungsstätten und in den Organisationen der Sozialen Arbeit schon lange: Man wolle wissen, was wirkt und wie man Probleme besser, schneller und ressourcenschonender angehen kann. Die Grundlagenforschung kam jedoch in der Sozialen Arbeit oft zu kurz, was großteils dem Status und der Ausstattung der Fachhochschulen geschuldet war.

Die Soziale Arbeit sei eine komplexe und angewandte Disziplin mit einem doppelten Mandat, psychosoziale Lebensweisen und Lebenslagen mittels professioneller Methoden zu verstehen und zu verändern, schreiben Silke Birgitta Gahleitner und Ingrid Miethe in ihrem Einleitungsbeitrag zu dem Themenheft der Blätter der Wohlfahrtspflege. Für die Soziale Arbeit sei es deshalb von besonderer Bedeutung, Forschung konkret auf Individuen und Gruppen in ihren jeweiligen sozialen Kontexten - Institutionen, Organisationen, Gemeinwesen etc. - auszurichten. Die Einzelbeiträge der Ausgabe sind:
Forschung tut not

Wissen generieren für die Theorie und für die Praxis Sozialer Arbeit

Von Silke Gableitner und Ingrid Miethe

Soziale Arbeit braucht qualifizierte Grundlagenforschung

Die Stichworte lauten Finanzierung, Forscherpersönlichkeiten, Interdisziplinarität

Von Konrad Maier

Forschende Lehre, forschende Praxis

Qualitative Forschungsmethoden und die Praxis Sozialer Arbeit

Von Ingrid Miethe

Beobachten, hinhören, fragen

Lehrforschung in den modularisierten Studiengängen der Sozialen Arbeit

Von Christine Schönberger und Burkhard Hill

Der Fall entsteht im Gespräch

Theoriebildung narrativ reflexiver Beratung als Aufgabe Sozialer Arbeit

Von Ulrike Loch und Heidrun Schulze

Forschen im Grenzbereich

Hard-to-reach-Klienten als Zielgruppe in der Sozialarbeitsforschung - Beispiel wohnungslose Menschen

Von Susanne Gerull

Forschen für eine bessere Jugendhilfe

Kriterium muss die Orientierung am Blickwinkel der jungen Menschen sein

Von Silke Birgitta Gableitner

»Transkulturalität « als Leitlinie

Rekonstruktive Methoden für die interkulturelle Praxis in der Sozialen Arbeit

Von Cornelia Giebeler

Buchreihe informiert über »Rekonstruktive Forschung in der Sozialen Arbeit «

Von Sonja Kubisch

Forschung für die Praxis: drei Beispiele

Zwischen Wahrheit und Richtigkeit

Sozialpädagogische Forschung in der Dialektik objektiver und subjektiver Ansprüche

Von Bernd Birgmeier und Martin Stummbaum

Hilfen über Hürden

Auch Fachhochschulabsolventen der Sozialen Arbeit können promovieren

Von Rudolf Schmitt

Das Einzelheft der Blätter der Wohlfahrtspflege kostet 14,Euro zuzüglich Versandkosten. Bestelladresse: Nomos Verlagsgesellschaft, 76520 Baden-Baden, Telefon 0722 2104-39, Fax072212104-43, E-Mail hohmann@nomos.de, Internet http://www.blaetter-der-wohlfahrtspflege.de 